\title{
NEWS2 and laboratory predictors correlated with clinical deterioration in hospitalised patients with COVID-19
}

Gulsah Tuncer ${ }^{1}$, Serkan Surme ${ }^{1}$, Osman Faruk Bayramlar ${ }^{2}$, Hatice Kubra Karanalbant ${ }^{1}$, Betul Copur $^{1}$, Meltem Yazla ${ }^{1}$, Esra Zerdali ${ }^{1}$, Inci Yilmaz Nakir ${ }^{1}$, Ayse Kurt Cinar ${ }^{1}$, Ahmet Buyukyazgan ${ }^{1}$, Hatice Balli ${ }^{1}$, Yesim Kurekci ${ }^{1}$, Serap Simsek Yavuz ${ }^{3}$, Mehmet Mesut Sonmez ${ }^{4}$, Gonul Sengoz ${ }^{1}$, Filiz Pehlivanoglu ${ }^{1}$

${ }^{1}$ Department of Infectious Diseases and Clinical Microbiology, Haseki Training and Research Hospital, Istanbul, Turkey

${ }^{2}$ Department of Public Health, Bakirkoy District Health Directorate, Istanbul, Turkey

${ }^{3}$ Department of Infectious Diseases and Clinical Microbiology, Istanbul Faculty of Medicine, Istanbul University, Istanbul, Turkey

${ }^{4}$ Department of Orthopaedic Surgery and Traumatology, Haseki Training and Research Hospital, Istanbul, Turkey

Corresponding author: "Serkan Surme” Department of Infectious Diseases and Clinical Microbiology, Haseki Training and Research Hospital, 34096, Istanbul, Turkey

e-mail: serkansurme@ hotmail.com, phone number: 905558578517

Keywords: COVID-19; NEWS2; in-hospital mortality; procalcitonin; albumin; neutrophil/lymphocyte ratio

Running Title: NEWS2 and laboratory predictors for clinical deterioration in COVID-19

\section{ABSTRACT}

Background: We aimed to determine prognostic values of NEWS2 and laboratory parameters during the first week of COVID-19.

Methods: All adult patients who were hospitalized for a confirmed COVID-19 between the 11th of March and the 11th of May 2020 were retrospectively included. To evaluate the factors in 
prognosis which are admission to intensive care unit (ICU) and in-hospital death, univariate logistic regression analysis was performed at admission (D0), at day-3 (D3), day-5 (D5), and day-7 (D7). Additionally, receiver operating characteristic (ROC) analyses were performed.

Results: Overall, 611 patients were included. Clinical deterioration was observed in 79 (12.9\%) patients during hospitalisation, $36(5.9 \%)$ during the first three days, 54 (8.8\%) during the first five days, and $62(10.1 \%)$ during the first week of hospitalisation. Our results showed that NEWS2, procalcitonin, neutrophil/lymphocyte ratio (NLR), and albumin were the best predictors for clinical deterioration at D0, D3, D5, and D7. Procalcitonin had the highest odds ratio for clinical deterioration on all days in univariate analysis. ROC analyses showed that NEWS2 at D7, procalcitonin at D5, albumin at D7, and NLR at D5 had highest AUC values. Additionally, we detected a strong correlation between NEWS2 and laboratory parameters including neutrophil, lymphocyte, NLR, platelet/lymphocyte ratio, CRP, procalcitonin, ferritin, and urea on all days.

Conclusion: This study provides a list of several laboratory parameters correlated with NEWS2 and potential predictors for ICU admission or in-hospital death during the clinical course of COVID-19. Dynamic monitoring of NEWS2 and laboratory parameters is vital for improving clinical outcomes.

\section{INTRODUCTION}

The COVID-19 pandemic due to the SARS-CoV-2 virus causes high rates of mortality, morbidity, longer duration of hospitalization and increased need for intensive care unit (ICU) admission (1). Improving critical care patient flow is crucial for high quality care to severe cases. Therefore, we need to predict clinical deterioration in patients with COVID-19, in order to hospitalize the patients and admit to ICU, when necessary. The National Institute for Health and Care Excellence recommend The National Early Warning Score 2 (NEWS2) to predict the risk for clinical deterioration in patients with COVID-19 $(2,3)$. NEWS2 is a simple scoring system including physiological parameters and vital signs (respiratory rate, oxygen saturation, systolic blood pressure, heart rate, level of consciousness, temperature and supplemental oxygen dependency) used to predict the risk for acute deterioration including sepsis $(4,5)$. An increasing number of studies have assessed the parameters in NEWS2 score for severe COVID-19 illness 
(6). However there is a lack of knowledge about the predictive value of NEWS2, despite some studies focus on NEWS2 and related scores (3).

In this study, we aimed to determine prognostic value of NEWS2 and laboratory parameters in COVID-19 patients. Additionally, the correlation between NEWS2 and laboratory parameters at admission, D3, D5, and D7 during the clinical course of COVID-19 were evaluated.

\section{PATIENTS AND METHODS}

\subsection{Study Design and Patients}

In this retrospective and single-center study, all adult patients ( $\geq 18$ years old) who were hospitalized for a laboratory confirmed COVID-19 between the 9th of March and the 8th of May 2020 were included. SARS-CoV-2 testing was performed by real-time reverse transcriptionpolymerase chain reaction (RT-PCR) of samples collected by nasopharyngeal and/or oropharyngeal swabs.

Patients with COVID-19 requiring hospitalisation were included in the study. Outpatients and asymptomatic patientswere excluded. Also, we excluded patients if oropharyngeal or nasopharyngeal swab samples were repeatedly negative for SARS-CoV-2 by RT-PCR. Our primary outcome was the occurence of clinical deterioration defined as a composite of ICU admission during hospitalisation or in-hospital death.

\subsection{Data collection}

Epidemiological and demographic characteristics, clinical, laboratory, radiological findings, and outcomes were collected from medical records. Vital signs including respiratory rate, peripheral capillary oxygen saturation, heart rate, blood pressure, body temperature, and consciousness (Glasgow Coma Scale) were recorded. Laboratory parameters including albumin, C-reactive protein (CRP), procalcitonin, haemoglobin, hematocrit, neutrophil count, lymphocyte count,, platelet count, neutrophil/lymphocyte ratio (NLR), platelet/lymphocyte ratio (PLR), urea, ferritin, albumin, fibrinogen, d-dimer, aspartate aminotransferase (AST), and alanin aminotransferase (ALT) were included. The NEWS2 score was calculated.

\subsection{Statistical analysis}


Quantitative variables are expressed as mean \& standard deviation if they contain continuous and normal distributed data. If the data was not distributed normally, median and interquartile range (IQR) were used. If they contain categorical data, they are expressed as percentage (\%) and frequency (n). Comparison of qualitative variables was analyzed with Pearson Chi square test. The normal distribution questioning the necessity of using the parametric test was examined by Kolmogrov-Smirnov, Shapiro Wilk, Curtosis - Skewness Tests and Box Plot Distribution. When normally distributed data could not be determined and non-parametric tests and spearman correlation were used. Kruskal Wallis Test was used for the analysis of continuous and more than two independent non-parametric groups (Bonferroni correction was used when necessary) and Mann Whitney Test was used for post-hoc analysis. To evaluate the factors in prognosis which are admission ICU and in-hospital death, univariate logistic regression analysis was performed. Afterwards, these dependent groups were handled one by one, ROC curves were drawn and cut-off values, sensitive and specificity of cut-off values, and Area Under the Curve (AUC), were demonstrated. To predict clinical deterioration, the prognostic accuracy of NEWS2 and laboratory parameters was evaluated for:

1. NEWS2 and laboratory parameters associated with the clinical deterioration at D0

2. NEWS2 and laboratory parameters associated with the clinical deterioration at D3 after excluding from the analysis patients with clinical deterioration within the first three days of hospitalization.

3. NEWS2 and laboratory parameters associated with the clinical deterioration at D5 after excluding from the analysis patients with clinical deterioration within the first five days of hospitalization.

4. NEWS2 and laboratory parameters associated with the clinical deterioration at D7 after excluding from the analysis patients with clinical deterioration within the first seven days of hospitalization.

The results were evaluated in $95 \%$ confidence interval and statistical significance level was defined as $\mathrm{p}<0.05$. The analyzes were performed using IBM SPSS - 21 (Statistical Package for Social Sciences, Chicago, IL, USA)

\section{RESULTS}




\subsection{General Characteristics}

Overall, 611 patients were included. Of whom, $329(53.8 \%)$ were male, the mean age was $52.53 \pm 15.07$ years. 73 patients $(11.9 \%)$ were admitted to the ICU. In-hospital death occurred in $46(7.5 \%)$ patients. Clinical deterioration was observed in 79 patients (12.9\%) during hospitalisation, 36 (5.9\%), during the first three days, 54 (8.8\%) during the first five days, and 62 $(10.1 \%)$ during the first week of hospitalisation. NEWS2 was calculated at admission (D0), D3, D5 and D7 of hospitalization. Patients were stratified into three risk groups: low risk from 0 to 4; medium risk from 5 to 6 and high risk above 7. Of 611 patients, $505(82.7 \%)$ at D0, $411(91.9 \%)$ at D3, $375(92.2 \%)$ at D5, $284(93.8 \%)$ at D7 had a NEWS2 score $<7$. The median length of hospital stay was 8.9 days and 332 patients (54.3\%) who did not have fever and did not need oxygen in the last 48-72 hours and meet the criteria for home monitoring were discharged within the first 7 days. Characteristic of patient, NEWS2 value and NEWS2 class at D0, D3, D5 and D7, are represented in Supp. Table.

Suppl. Table. Characteristics, NEWS2 value anda class of patients hospitalized for COVID-19

\begin{tabular}{|l|c|}
\hline & Overall (n: 611) \\
\hline Age, mean \pm SD & $52.53 \pm 15.07$ \\
\hline Sex & \\
Male, $\mathrm{n}(\%)$ & $329(53.8 \%)$ \\
\hline Serious COVID-19 risk factors, $\mathbf{n}(\%)$ & $131(21.3 \%)$ \\
\hline Age $>$ 65 years old, $\mathrm{n}(\%)$ & $170(27.6 \%)$ \\
\hline Diabetes mellitus, $\mathrm{n}(\%)$ & $192(31.2 \%)$ \\
\hline Hypertension & $13(2.1 \%)$ \\
\hline Cardiovascular diseases, $\mathrm{n}(\%)$ & $4(0.6 \%)$ \\
\hline Cerebrovascular diseases, $\mathrm{n}(\%)$ & $34(5.5 \%)$ \\
\hline Chronic kidney failure, $\mathrm{n}(\%)$ & $10(1.2 \%)$ \\
\hline Malignancy, $\mathrm{n}(\%)$ & $68(11.1 \%)$ \\
\hline Chronic respiratory diseases, $\mathrm{n}(\%)$ & $1(0.2 \%)$ \\
\hline HIV/AIDS, $\mathrm{n}(\%)$ & $5(0.8 \%)$ \\
\hline Severe chronic liver disease, $\mathrm{n}(\%)$ & $4(0.6 \%)$ \\
\hline Rheumatological disease, $\mathrm{n}(\%)$ & $3(0.5 \%)$ \\
\hline Dementia, $\mathrm{n}(\%)$ & 0 \\
\hline Malnutrition, $\mathrm{n}(\%)$ & $5(0.8 \%)$ \\
\hline Neurological disorder, $\mathrm{n}(\%)$ & $3(0.5 \%)$ \\
\hline Chronic hematological disease, $\mathrm{n}(\%)$ & $6(1 \%)$ \\
\hline Immunodepression, $\mathrm{n}(\%)$ & \\
\hline
\end{tabular}




\begin{tabular}{|c|c|}
\hline Current smoker & $65(15.6 \%)$ \\
\hline \multicolumn{2}{|l|}{ Clinical signs before admission } \\
\hline Fever, n (\%) & $342(55.6 \%)$ \\
\hline Cough, n (\%) & $473(76.8 \%)$ \\
\hline Dyspnea, n (\%) & $251(40.7 \%)$ \\
\hline Rhinorrhea and/or nasal congestion & $7(1.1 \%)$ \\
\hline Chest pain or pressure & $19(3.1 \%)$ \\
\hline Myalgias & $76(12.3 \%)$ \\
\hline Arthralgia & $21(3.4 \%)$ \\
\hline Fatigue & $215(34.9 \%)$ \\
\hline Sore throat & $53(8.6 \%)$ \\
\hline Stomachache & $11(1.8 \%)$ \\
\hline Nausea/Vomiting & $40(6.5 \%)$ \\
\hline Diarrhea & $25(4.1 \%)$ \\
\hline Confusion, n (\%) & 0 \\
\hline Ageusia & $4(0.6 \%)$ \\
\hline Anosmia, n (\%) & $3(0.5 \%)$ \\
\hline Systolic blood pressure median (IQR) & $120 / 20$ \\
\hline Diastolic blood pressure median (IQR) & $70 / 10$ \\
\hline Temperature median (IQR) & $36.6 / 0.8$ \\
\hline Respiration rate median (IQR) & $21 / 2.8$ \\
\hline Heart rate median (IQR) & $88 / 15$ \\
\hline Oxygen saturation median (IQR) & $94 / 4$ \\
\hline \multicolumn{2}{|l|}{ Laboratory abnormalities at admission } \\
\hline Leukocyte count/uL, median (IQR) & $5,950 / 3,050$ \\
\hline Neutrophil count/uL, median (IQR) & $3,820 / 2,590$ \\
\hline Lymphocyte count/uL, median (IQR) & $1,350 / 820$ \\
\hline Neutrophil/lymphocyte ratio & $2.87 / 2.41$ \\
\hline Platelet/lymphocyte ratio & $143.5 / 85.7$ \\
\hline Platelet count/uL, median (IQR) & $194,000 / 84,750$ \\
\hline Haemoglobin, g/dL, median (IQR) & $13 / 2$ \\
\hline Hematocrit, \%, median (IQR) & $39 / 5.4$ \\
\hline Urea, mmol/L, median (IQR) & $28 / 13$ \\
\hline Creatinine, $\mathrm{mg} / \mathrm{dL}$, median (IQR) & $109 / 146$ \\
\hline Aspartate aminotransferase (AST), UI/L, median (IQR) & $31 / 19$ \\
\hline Alanine aminotransferase (ALT), UI/L, median (IQR) & $23 / 18$ \\
\hline Lactate dehydrogenase (LDH), UI/L, median (IQR) & $268 / 127$ \\
\hline Creatine phosphokinase (CPK), UI/L, median (IQR) & $99 / 146$ \\
\hline Albumin, g/L, median (IQR) & $37 / 5$ \\
\hline Ferritin, ng/L, median (IQR) & $155.5 / 243$ \\
\hline C-reactive protein $(\mathrm{CRP}), \mathrm{mg} / \mathrm{L}$, median $(\mathrm{IQR})$ & $42.8 / 74.5$ \\
\hline Troponin, ng/mL, median (IQR) & $4.1 / 5.5$ \\
\hline Procalcitoin, ng/mL, median (IQR) & $0.18 / 0.07$ \\
\hline D-dimer, mg/L, median (IQR) & $0.64 / 0.69$ \\
\hline CT & \\
\hline
\end{tabular}




\begin{tabular}{|c|c|}
\hline Unilateral & $35(6 \%)$ \\
\hline Bilateral & $551(94 \%)$ \\
\hline NEWS2 at D0 & Overall (n: $)$ \\
\hline Median (min, max) & $4(0-14)$ \\
\hline Class I & $352(57.6 \%)$ \\
\hline Class II & $153(25 \%)$ \\
\hline Class III & $106(17.3 \%)$ \\
\hline NEWS2 at D3 & Overall (n: $)$ \\
\hline Median (min, max) & $3(0-14)$ \\
\hline Class I & $322(72 \%)$ \\
\hline Class II & $89(19.9 \%)$ \\
\hline Class III & $36(8.1 \%)$ \\
\hline NEWS2 at D5 & Overall (n: $)$ \\
\hline Median (min, max) & $3(0-12)$ \\
\hline Class I & $308(75.7 \%)$ \\
\hline Class II & $67(16.5 \%)$ \\
\hline Class III & $32(7.9 \%)$ \\
\hline NEWS2 at D7 & Overall (n: $)$ \\
\hline Median (min, max) & $3(0-9)$ \\
\hline Class I & $239(78.9 \%)$ \\
\hline Class II & $45(14.9 \%)$ \\
\hline Class III & $19(6.3)$ \\
\hline
\end{tabular}

Class I: 0-4 points

Class II: 5-6 points

Class III: 7-20 points

\subsection{ICU admission or in-hospital mortality}

The parameters associated with admission ICU and in-hospital death at D0, D3, D5, and D7 were NEWS2, lymphocyte count, neutrophil count, platelet count, NLR, PLR, CRP, procalcitonin, ddimer, troponin, AST, urea, LDH, and albumin. The median and IQR values of the laboratory parameters and are available in Table-1.

Table 1. Median and interquartile range (IQR) values of the parameters

\begin{tabular}{|c|c|c|c|c|c|c|c|c|}
\hline \multirow{2}{*}{ Parameters } & \multicolumn{2}{|c|}{ D0 } & \multicolumn{2}{c|}{ D3 } & \multicolumn{2}{c|}{ D5 } & \multicolumn{2}{c|}{ D7 } \\
\cline { 2 - 10 } & \multicolumn{2}{|c|}{ Prognosis } & \multicolumn{2}{c|}{ Prognosis } & \multicolumn{2}{c|}{ Prognosis } & \multicolumn{2}{c|}{ Prognosis } \\
\hline NEWS2 & Poor & Good & Poor & Good & Poor & Good & Poor & Good \\
\hline IQR & 4 & 3 & 3 & 2 & 3 & 3 & 4 & 2 \\
\hline Median & 6 & 4 & 5 & 3 & 6 & 3 & 7 & 3 \\
\hline p value & \multicolumn{2}{|c|}{0.001} & \multicolumn{2}{|c|}{0.001} & \multicolumn{2}{|c|}{0.001} & \multicolumn{2}{|c|}{0.001} \\
\hline Leukocyte count & Poor & Good & Poor & Good & Poor & Good & Poor & Good \\
\hline IQR & 4020 & 2990 & 6745 & 2628 & 6383 & 2390 & 4223 & 2200 \\
\hline Median & 6075 & 5930 & 7905 & 5800 & 7910 & 5880 & 7620 & 6220 \\
\hline
\end{tabular}




\begin{tabular}{|c|c|c|c|c|c|c|c|c|}
\hline $\mathrm{p}$ value & \multicolumn{2}{|c|}{0.26} & \multicolumn{2}{|c|}{0.001} & \multicolumn{2}{|c|}{0.01} & \multicolumn{2}{|c|}{0.04} \\
\hline Lymphocyte count & Poor & Good & Poor & Good & Poor & Good & Poor & Good \\
\hline IQR & 538 & 810 & 508 & 750 & 295 & 793 & 786 & 1600 \\
\hline Median & 1030 & 1390 & 805 & 1420 & 710 & 1505 & 685 & 1470 \\
\hline $\mathrm{p}$ value & \multicolumn{2}{|c|}{0.001} & \multicolumn{2}{|c|}{0.001} & \multicolumn{2}{|c|}{0.001} & \multicolumn{2}{|c|}{0.001} \\
\hline Neutrophil count & Poor & Good & Poor & Good & Poor & Good & Poor & Good \\
\hline$\overline{\mathrm{IQR}}$ & 4013 & 2410 & 5830 & 2038 & 6750 & 1910 & 3958 & 1810 \\
\hline Median & 4645 & 3710 & 6565 & 3505 & 6520 & 3680 & 6475 & 3800 \\
\hline $\mathrm{p}$ value & \multicolumn{2}{|c|}{0.001} & \multicolumn{2}{|c|}{0.001} & \multicolumn{2}{|c|}{0.001} & \multicolumn{2}{|c|}{0.001} \\
\hline Platelet count $\times 10^{3}$ & Poor & Good & Poor & Good & Poor & Good & Poor & Good \\
\hline IQR & 83 & 82.75 & 132.5 & 120.75 & 73 & 163.5 & 74.5 & 160 \\
\hline Median & 175 & 197 & 200.5 & 237 & 206.5 & 281 & 229.5 & 329 \\
\hline $\mathrm{p}$ value & \multicolumn{2}{|c|}{0.04} & \multicolumn{2}{|c|}{0.01} & \multicolumn{2}{|c|}{0.001} & \multicolumn{2}{|c|}{0.01} \\
\hline NLR & Poor & Good & Poor & Good & Poor & Good & Poor & Good \\
\hline IQR & 4.54 & 2.11 & 8.28 & 1.84 & 7.36 & 1.86 & 10.65 & 1.64 \\
\hline Median & 4.83 & 2.7 & 6.6 & 2.35 & 8.5 & 2.29 & 7.77 & 2.6 \\
\hline $\mathrm{p}$ value & \multicolumn{2}{|c|}{0.001} & \multicolumn{2}{|c|}{0.001} & & & & \\
\hline PLR & Poor & Good & Poor & Good & Poor & Good & Poor & Good \\
\hline IQR & 129.8 & 78.2 & 173.1 & 101.6 & 154.9 & 119.8 & 280.8 & 135.2 \\
\hline Median & 180.3 & 141.5 & 250.8 & 162 & 278.3 & 180.2 & 341.3 & 223.3 \\
\hline $\mathrm{p}$ value & & & & & & & & \\
\hline CRP & Poor & Good & Poor & Good & Poor & Good & Poor & Good \\
\hline IQR & 99.8 & 65 & 139 & 60 & 115.3 & 50.3 & 139.6 & 48.6 \\
\hline Median & 110.5 & 33.1 & 127 & 29.8 & 183 & 24 & 142.5 & 19 \\
\hline $\mathrm{p}$ value & & & & & & & & \\
\hline Procalcitonin & Poor & Good & Poor & Good & Poor & Good & Poor & Good \\
\hline IQR & 0.64 & 0.05 & 0.79 & 0.05 & 1.08 & 0.03 & 0.88 & 0.05 \\
\hline Median & 0.19 & 0.04 & 0.45 & 0.04 & 0.58 & 0.04 & 0.20 & 0.05 \\
\hline $\mathrm{p}$ value & & & & & & & & \\
\hline D-dimer & Poor & Good & Poor & Good & Poor & Good & Poor & Good \\
\hline IQR & 1.06 & 0.64 & 3.82 & 0.76 & 5.97 & 0.95 & 4.47 & 1.11 \\
\hline Median & 0.8 & 0.63 & 1.4 & 0.8 & 1.4 & 1 & 2.7 & 1.3 \\
\hline $\mathrm{p}$ value & & & & & & & & \\
\hline Ferritin & Poor & Good & Poor & Good & Poor & Good & Poor & Good \\
\hline IQR & 416 & 218 & 840 & 174 & 4008 & 241 & 1631 & 190 \\
\hline Median & 234 & 146 & 583 & 171 & 500 & 203 & 499 & 208 \\
\hline $\mathrm{p}$ value & & & & & & & & \\
\hline Troponin & Poor & Good & Poor & Good & Poor & Good & Poor & Good \\
\hline IQR & 39 & 4.40 & 29.80 & 3.40 & 35.85 & 2.92 & 44.90 & 4.00 \\
\hline Median & 14.6 & 3.9 & 12.5 & 3.2 & 27.5 & 3.3 & 19.1 & 4 \\
\hline $\mathrm{p}$ value & & & & & & & & \\
\hline AST & Poor & Good & Poor & Good & Poor & Good & Poor & Good \\
\hline Mean & 23 & 19 & 41 & 24 & 24 & 21 & 27 & 22 \\
\hline Median & 36.5 & 30 & 51 & 31 & 48 & 36 & 46.5 & 36 \\
\hline $\mathrm{p}$ value & & & & & & & & \\
\hline ALT & Poor & Good & Poor & Good & Poor & Good & Poor & Good \\
\hline IQR & 13 & 19 & 34 & 26 & 28 & 32 & 30 & 37 \\
\hline Median & 22.5 & 23 & 27 & 26 & 30.5 & 32 & 28.5 & 35.5 \\
\hline $\mathrm{p}$ value & & & & & & & & \\
\hline Creatinine & Poor & Good & Poor & Good & Poor & Good & Poor & Good \\
\hline IQR & 0.52 & 0.30 & 0.80 & 0.25 & 1.14 & 0.25 & 0.97 & 0.20 \\
\hline Median & 0.98 & 0.72 & 0.86 & 0.7 & 0.9 & 0.68 & 0.9 & 0.7 \\
\hline $\mathrm{p}$ value & & & & & & & & \\
\hline
\end{tabular}




\begin{tabular}{|c|c|c|c|c|c|c|c|c|}
\hline Urea & Poor & Good & Poor & Good & Poor & Good & Poor & Good \\
\hline IQR & 30 & 12 & 34 & 12 & 46 & 12 & 51 & 13 \\
\hline Median & 39.4 & 27 & 43.7 & 25 & 43.5 & 24 & 33 & 25.8 \\
\hline $\mathrm{p}$ value & \multicolumn{2}{|c|}{0.001} & \multicolumn{2}{|c|}{0.001} & \multicolumn{2}{|c|}{0.001} & \multicolumn{2}{|c|}{0.01} \\
\hline LDH & Poor & Good & Poor & Good & Poor & Good & Poor & Good \\
\hline IQR & 197 & 114 & 250 & 137 & 151 & 128 & 196 & 138 \\
\hline Median & 336 & 262 & 468 & 272 & 513 & 281 & 485 & 289 \\
\hline $\mathrm{p}$ value & \multicolumn{2}{|c|}{0.001} & \multicolumn{2}{|c|}{0.001} & \multicolumn{2}{|c|}{0.001} & \multicolumn{2}{|c|}{0.001} \\
\hline CPK & Poor & Good & Poor & Good & Poor & Good & Poor & Good \\
\hline IQR & 210 & 124 & 360 & 80 & 263 & 58 & 457 & 55 \\
\hline Median & 170 & 102 & 181 & 72 & 124.5 & 63 & 161.5 & 57 \\
\hline $\mathrm{p}$ value & \multicolumn{2}{|c|}{0.02} & \multicolumn{2}{|c|}{0.001} & \multicolumn{2}{|c|}{0.08} & \multicolumn{2}{|c|}{0.01} \\
\hline Albumin & Poor & Good & Poor & Good & Poor & Good & Poor & Good \\
\hline IQR & 8 & 5 & 6 & 5 & 7 & 5 & 9 & 4 \\
\hline Median & 33 & 37 & 31 & 36.5 & 27.5 & 35 & 30 & 34 \\
\hline $\mathrm{p}$ value & \multicolumn{2}{|c|}{0.001} & \multicolumn{2}{|c|}{0.001} & \multicolumn{2}{|c|}{0.001} & \multicolumn{2}{|c|}{0.001} \\
\hline Haemoglobin & Poor & Good & Poor & Good & Poor & Good & Poor & Good \\
\hline IQR & 2.4 & 2.0 & 2.5 & 2.2 & 2.0 & 1.9 & 2.7 & 2.0 \\
\hline Median & 13 & 13 & 11.95 & 12.6 & 11.25 & 12.4 & 11.7 & 12.5 \\
\hline $\mathrm{p}$ value & \multicolumn{2}{|c|}{0.43} & \multicolumn{2}{|c|}{0.01} & \multicolumn{2}{|c|}{0.001} & \multicolumn{2}{|c|}{0.09} \\
\hline Hematocrit & Poor & Good & Poor & Good & Poor & Good & Poor & Good \\
\hline IQR & 7.1 & 5.1 & 5.7 & 5.2 & 4.8 & 6.0 & 6.1 & 5.8 \\
\hline Median & 38.9 & 39 & 36.05 & 37.7 & 34.3 & 37.1 & 35.6 & 36.7 \\
\hline $\mathrm{p}$ value & \multicolumn{2}{|c|}{0.24} & \multicolumn{2}{|c|}{0.01} & \multicolumn{2}{|c|}{0.001} & \multicolumn{2}{|c|}{0.16} \\
\hline
\end{tabular}

(D0: Admission, D3: Day-3, D5: Day-5, D7: Day-7, IQR: Interquartile Range, NLR: Neutrophil/lymphocyte ratio, PLR: Platelet/lymphocyte ratio, AST: Aspartate aminotransferase, ALT: Alanine aminotransferase, LDH; Lactate dehydrogenase, CPK: Creatine phosphokinase, CRP: C-reactive protein)

\subsection{Univariate analysis}

In univariate analysis, among parameters associated with ICU admission or in-hospital death at D0, D3, D5, and D7, best predictors were NEWS2, procalcitonin, NLR, and albumin. Additionally, d-dimer (at D0, D3, and D7) and haemoglobin (at D3 and D5) were valuable predictors in univariate analysis (Table-2).

Table 2. Univariate analysis of parameters associated with ICU admission and in-hospital death

\begin{tabular}{|l|c|c|c|}
\hline Parameter & p value & Odds ratio (OR) & 95\% CI \\
\hline NEWS2 at D0 & $<0.001$ & 1.386 & $1.255-1.530$ \\
\hline NEWS2 at D3 & $<0.001$ & 1.709 & $1.463-1.998$ \\
\hline NEWS2 at D5 & $<0.001$ & 1.933 & $1.564-2.389$ \\
\hline NEWS2 at D7 & $<0.001$ & 2.030 & $1.560-2.642$ \\
\hline Procalcitonin at D0 & $<0.001$ & 6.486 & $2.444-17.212$ \\
\hline Procalcitonin at D3 & $<0.001$ & 3.244 & $1.615-6.519$ \\
\hline Procalcitonin at D5 & $<0.001$ & 15.087 & $1.403-162.365$ \\
\hline Procalcitonin at D7 & 0.001 & 1.377 & $0.771-2.491$ \\
\hline
\end{tabular}


medRxiv preprint doi: https://doi.org/10.1101/2021.01.17.21249878; this version posted January 20, 2021. The copyright holder for this preprint (which was not certified by peer review) is the author/funder, who has granted medRxiv a license to display the preprint in perpetuity.

It is made available under a CC-BY-NC-ND 4.0 International license .

\begin{tabular}{|l|c|c|c|}
\hline NLR at D0 & $<0.001$ & 1.286 & $1.190-1.390$ \\
\hline NLR at D3 & $<0.001$ & 1.754 & $1.522-2.021$ \\
\hline NLR at D5 & $<0.001$ & 1.806 & $1.495-2.180$ \\
\hline NLR at D7 & $<0.001$ & 1.332 & $1.168-1.520$ \\
\hline Albumin at D0 & $<0.001$ & 0.782 & $0.714-0.856$ \\
\hline Albumin at D3 & $<0.001$ & 0.699 & $0.627-0.779$ \\
\hline Albumin at D5 & $<0.001$ & 0.752 & $0.664-0.851$ \\
\hline Albumin at D7 & $<0.001$ & 0.679 & $0.547-0.843$ \\
\hline Haemoglobin at D3 & 0.003 & 0.779 & $0.661-0.919$ \\
\hline Haemoglobin at D5 & $<0.001$ & 0.615 & $0.470-0.804$ \\
\hline D-dimer at D0 & 0.001 & 1.123 & $1.046-1.206$ \\
\hline D-dimer at D3 & 0.002 & 1.209 & $1.071-1.365$ \\
\hline D-dimer at D7 & 0.045 & 1.217 & $1.004-1.476$ \\
\hline
\end{tabular}

(D0: Admission, D3: Day-3, D5: Day-5, D7: Day-7, NLR: Neutrophil/lymphocyte ratio, PLR: Platelet/lymphocyte ratio AST: Aspartate aminotransferase, ALT: Alanine aminotransferase, LDH; Lactate dehydrogenase, CPK: Creatine phosphokinase, CRP: C-reactive protein)

\subsection{Correlation}

Laboratory parameters correlated with NEWS2 at D0, D3, D5, and D7 were lymphocyte count, neutrophil count, NLR, PLR, CRP, procalcitonin, ferritin and urea (Suppl. Fig. 1. and Suppl. Fig.

2.)

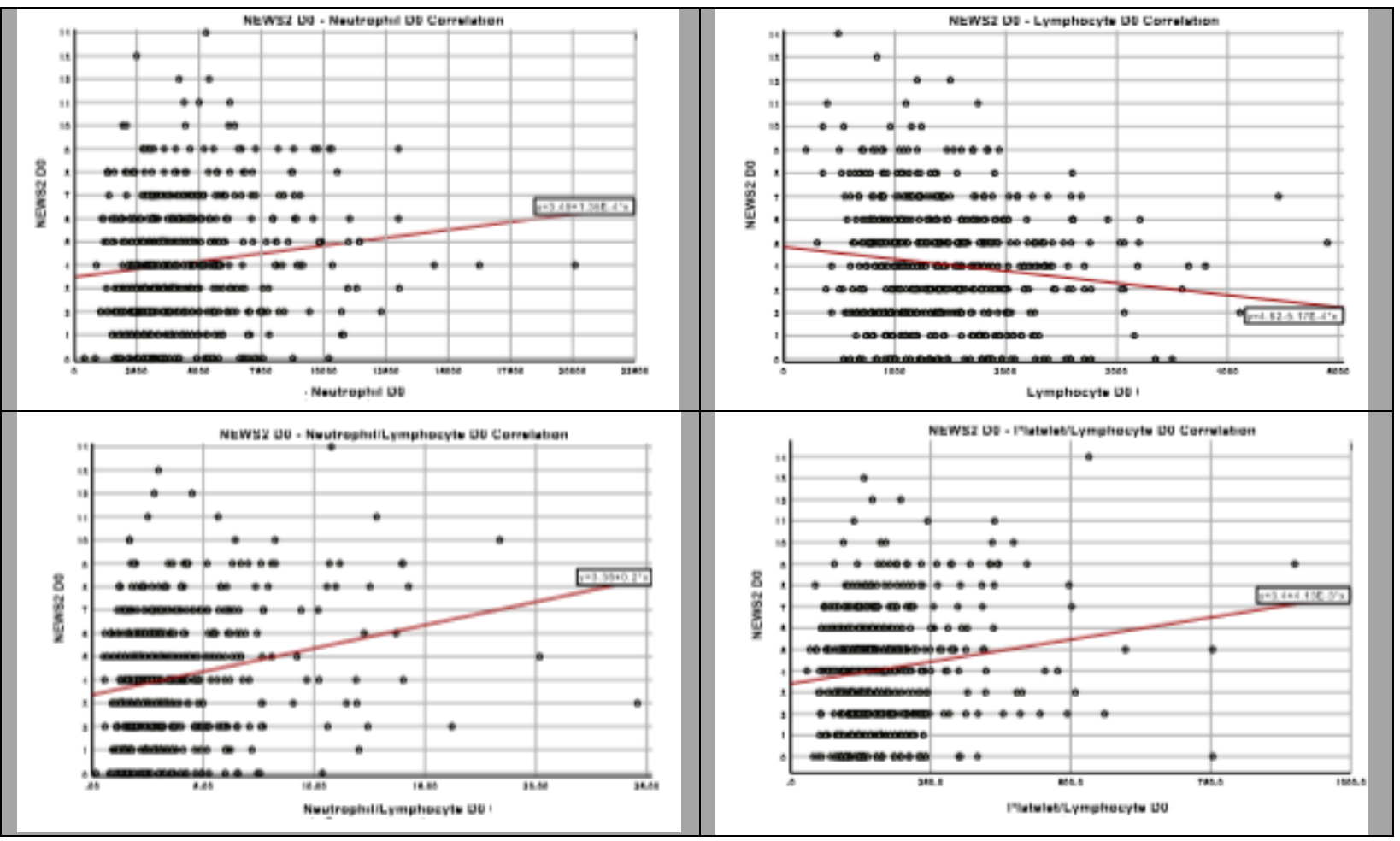




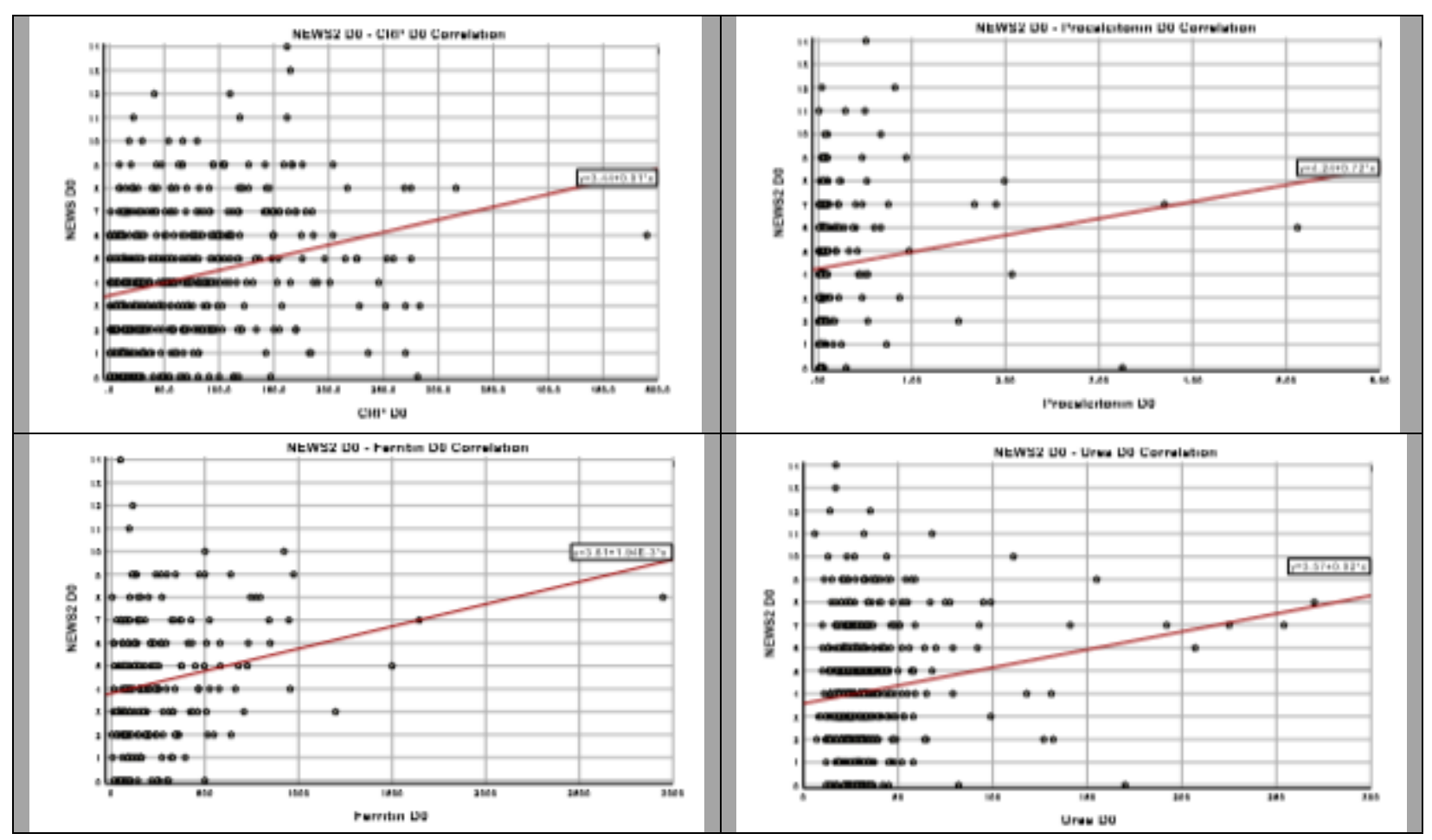

Supp. Figure 1. Correlation between NEWS2 and lymphocyte count, neutrophil count, NLR, PLR, CRP, procalcitonin, ferritin and urea at D0
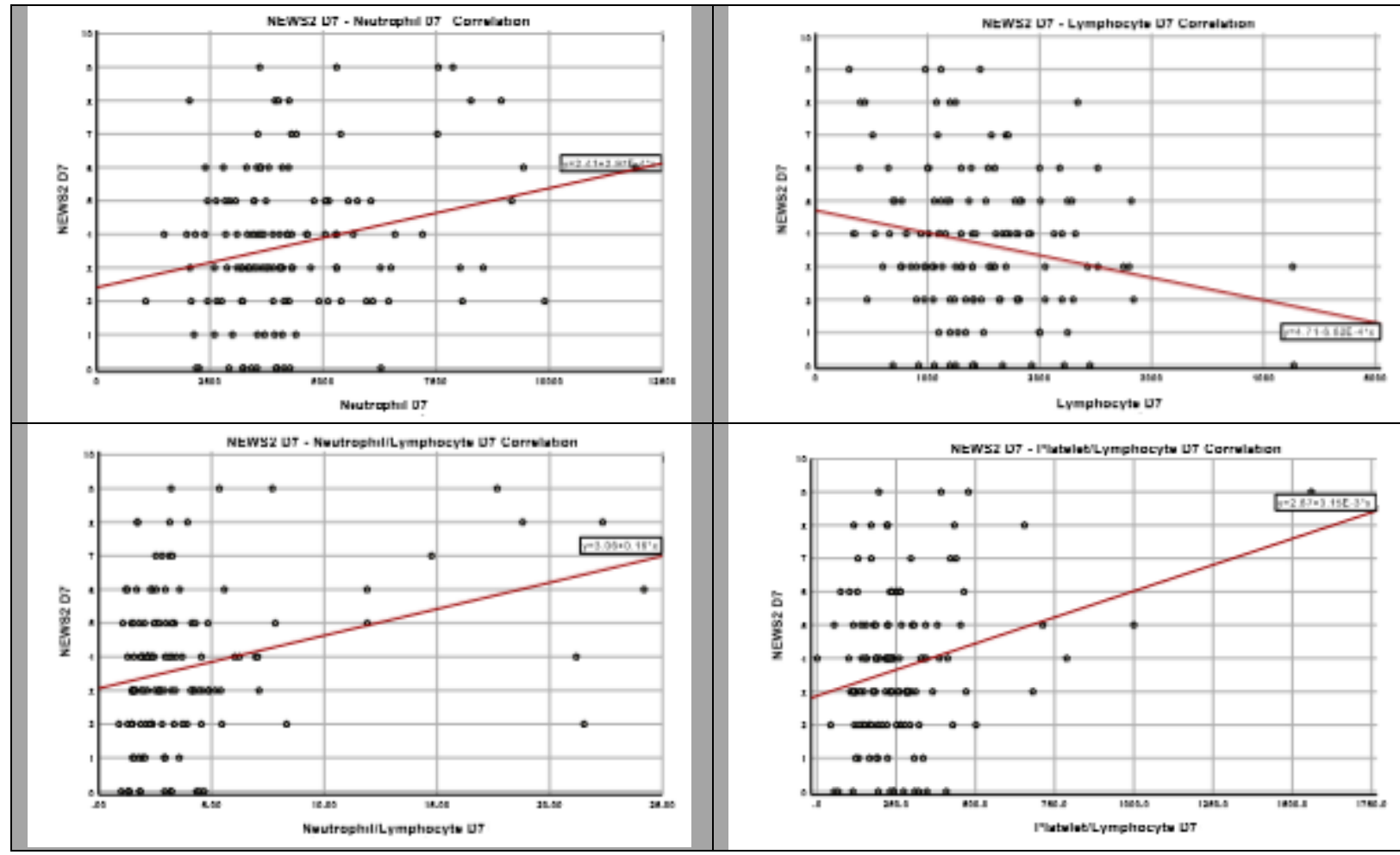


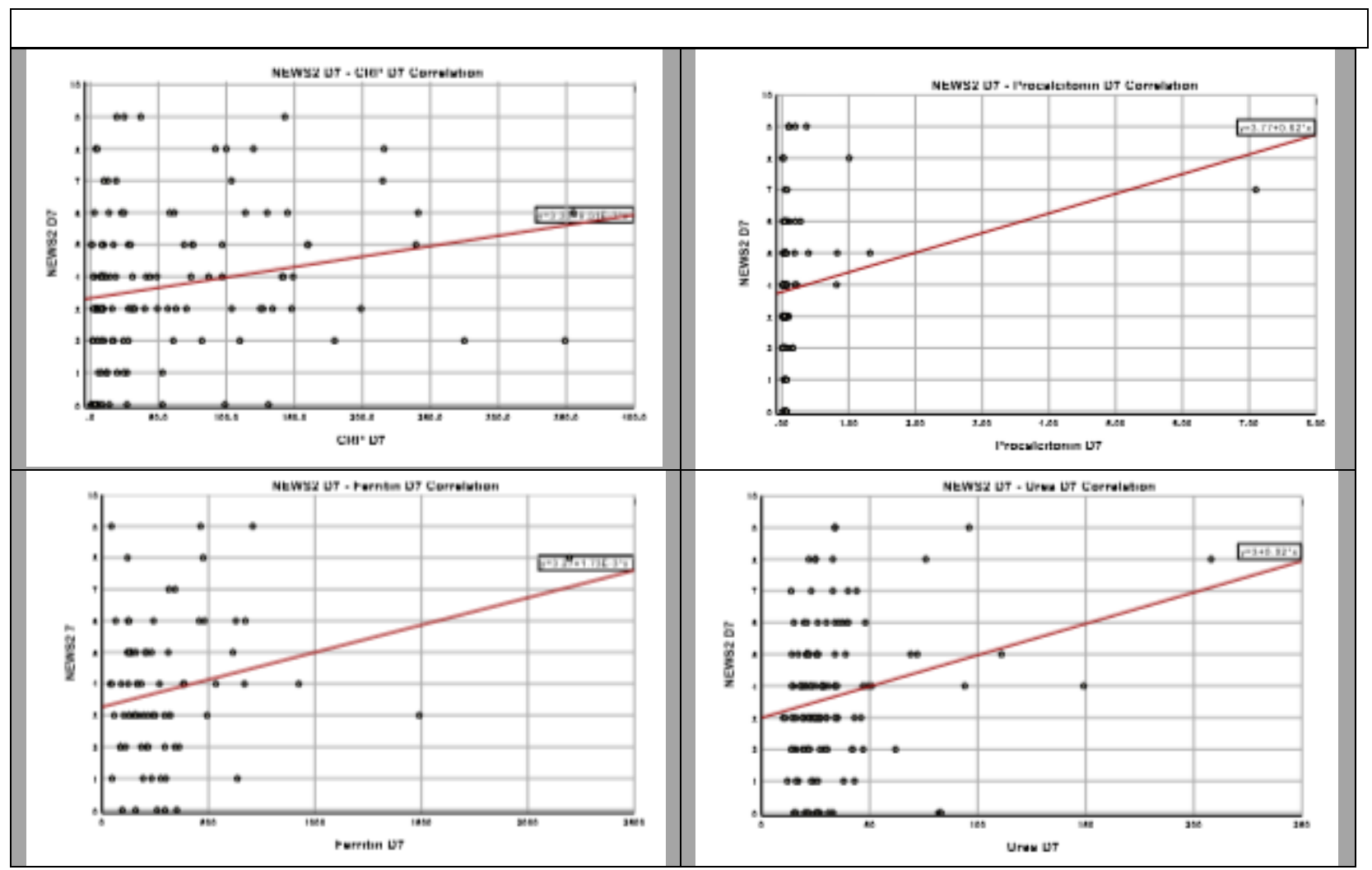

Supp Figure 2. Correlation between NEWS2 and lymphocyte count, neutrophil count, NLR, PLR, CRP, procalcitonin, ferritin and urea at D7

\subsection{ROC Curves}

ROC curves of NEWS2 at D0, D3, D5 and D7 to predict clinical deterioration are shown in Fig. 1. At D0, AUC curve was 0.726 (95\% CI 0.669- 0.784), with the best cut off of 4.5, sensitivity of $71.1 \%$ and specificity of $61.7 \%$ (p<0.001). At D3, AUC curve was 0.798 (95\% CI 0.740-0.856), with the best cut-off of 4.5 , sensitivity of \%69.6, and specificity of $\% 78$ ( $\mathrm{p}<0.001)$. At D5, AUC curve was 0.833 (95\% CI $0.755-0.911)$, with the best cut-off of 4.5 , sensitivity of $72.4 \%$ and specificity of $79.4 \%$ ( $\mathrm{p}<0.001)$. At D7, AUC curve was 0.842 (95\% CI 0.739-0.945), with the best cut-off of 3.5 , sensitivity of $88.2 \%$ and specificity of $64.7 \%(\mathrm{p}<0.001)$. 


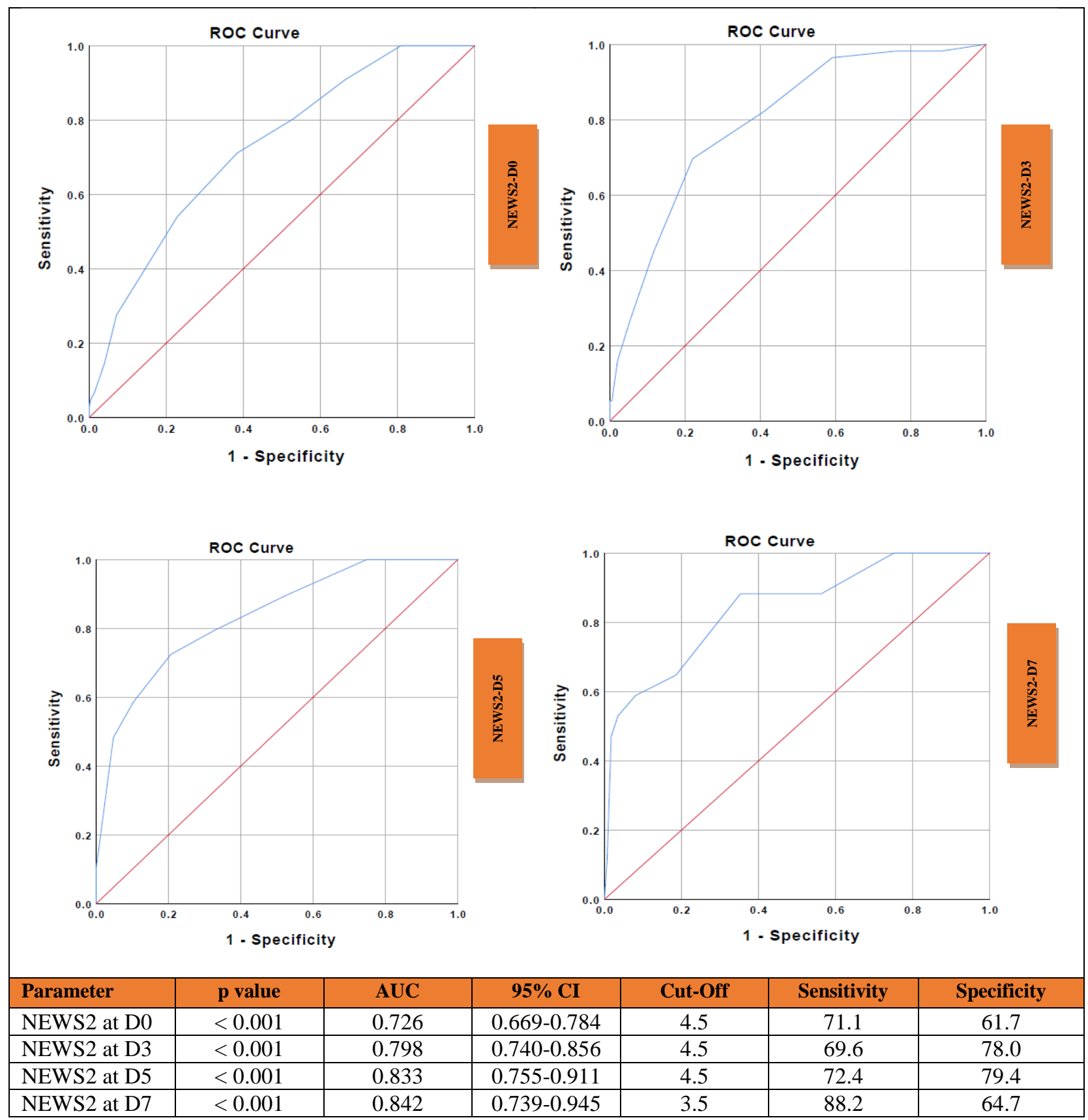

Figure 1. Receiver Operating Characteristic (ROC) curve and performance value for the best cut off for: A: NEWS2 at admission using clinical deterioration B: NEWS2 at D3 using clinical deterioration C: NEWS2 at D5 using clinical deterioration D: NEWS2 at D7 using clinical deterioration

ROC curves of procalcitonin at D0, D3, D5 and D7 to predict clinical deterioration are shown in Fig. 2. At D0, AUC curve was 0.824 (95\% CI 0.755- 0.893), with the best cut-off of 0.065, sensitivity of $81.1 \%$ and specificity of $67.9 \%$ ( $\mathrm{p}<0.001)$. At D3, AUC curve was 0.896 (95\% CI 0.837- 0.956 ), with the best cut-off of 0.125 , sensitivity of $80.6 \%$ and specificity of $89.3 \%$ ( $\mathrm{p}<0.001)$. At D5, AUC curve was 0.967 (95\% CI 0.928-1.000), with the best cut-off of 0.155, 
medRxiv preprint doi: https://doi.org/10.1101/2021.01.17.21249878; this version posted January 20, 2021. The copyright holder for this preprint (which was not certified by peer review) is the author/funder, who has granted medRxiv a license to display the preprint in perpetuity.

It is made available under a CC-BY-NC-ND 4.0 International license .

sensitivity of $87.5 \%$ and specificity of $96.7 \%$ (p<0.001). At D7, AUC curve was 0.823 (95\% CI 0.609-1.000), with the best cut-off of 0.120 , sensitivity of $85.7 \%$ and specificity of $88.2 \%$ $(\mathrm{p}<0.004)$.

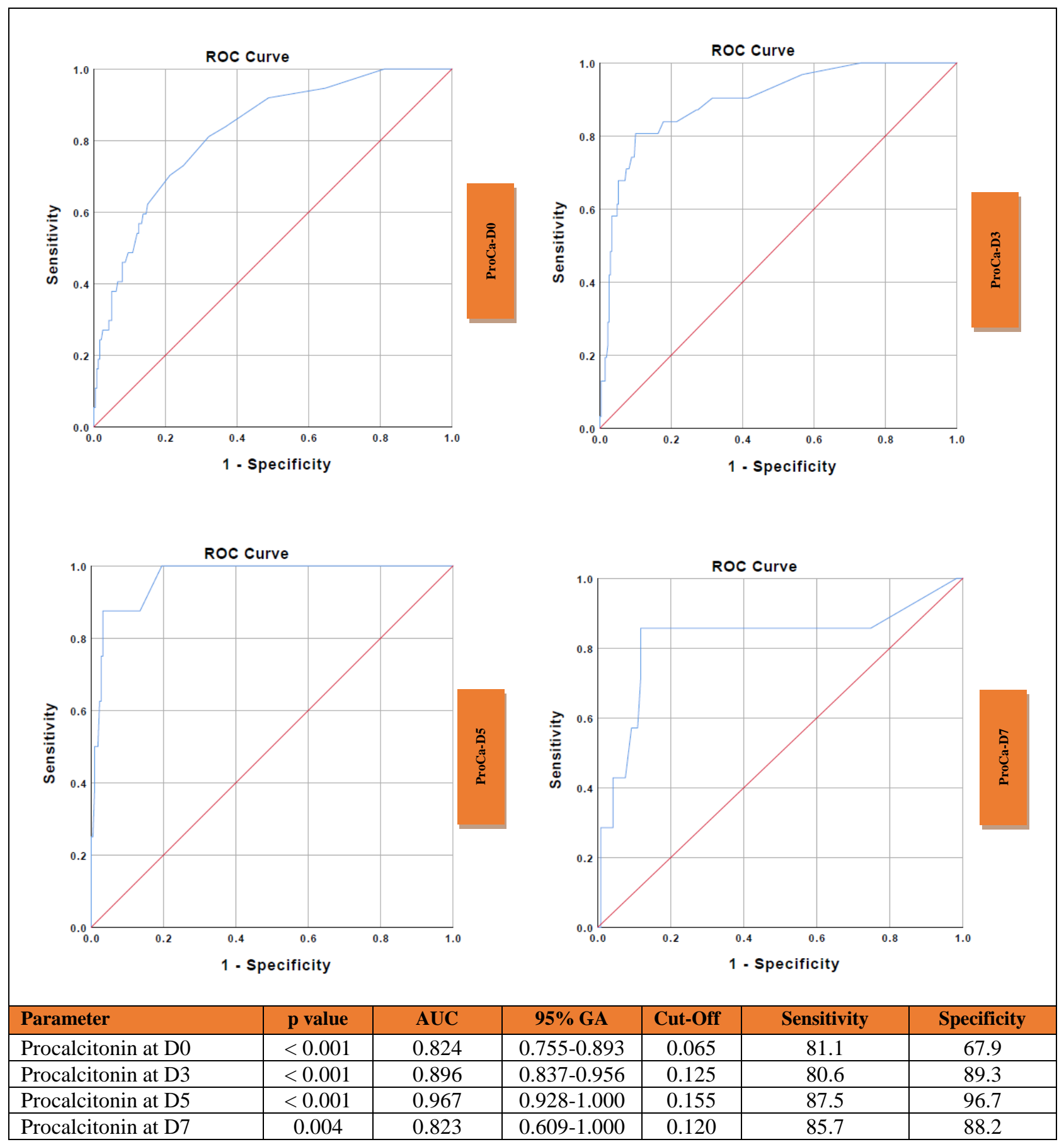

Figure 2. Receiver Operating Characteristic (ROC) curve and performance value for the best cut off for: A: procalcitonin at admission using clinical deterioration. B: procalcitonin at D3 using clinical deterioration. C: procalcitonin at D5 using clinical deterioration. D: procalcitonin at D7 using clinical deterioration. 
medRxiv preprint doi: https://doi.org/10.1101/2021.01.17.21249878; this version posted January 20, 2021. The copyright holder for this preprint (which was not certified by peer review) is the author/funder, who has granted medRxiv a license to display the preprint in perpetuity.

It is made available under a CC-BY-NC-ND 4.0 International license .

ROC curves of albumin at D0, D3, D5 and D7 to predict clinical deterioration are shown in Fig 3. At D0, AUC curve was 0.746 (95\% CI 0.656-0.835), with the best cut-off of 35.5, sensitivity of $69.2 \%$ and specificity of $66.7 \%(\mathrm{p}<0.001)$. At D3, AUC curve was 0.868 (95\% CI 0.8160.919), with the best cut-off of 33.5, sensitivity of $79.8 \%$ and specificity of $78.3 \%$ (p <0.001). At D5, AUC curve was 0.887 (95\% CI 0.814-0.960), with the best cut-off of 31.5, sensitivity of $89.3 \%$ and specificity of $70 \%$ ( $\mathrm{p}<0.001)$. At D7, AUC curve was 0.896 (95\% CI 0.813-0.979), with the best cut-off of 32.5 , sensitivity of $78.9 \%$ and specificity of $88.9 \%(\mathrm{p}<0.001)$.

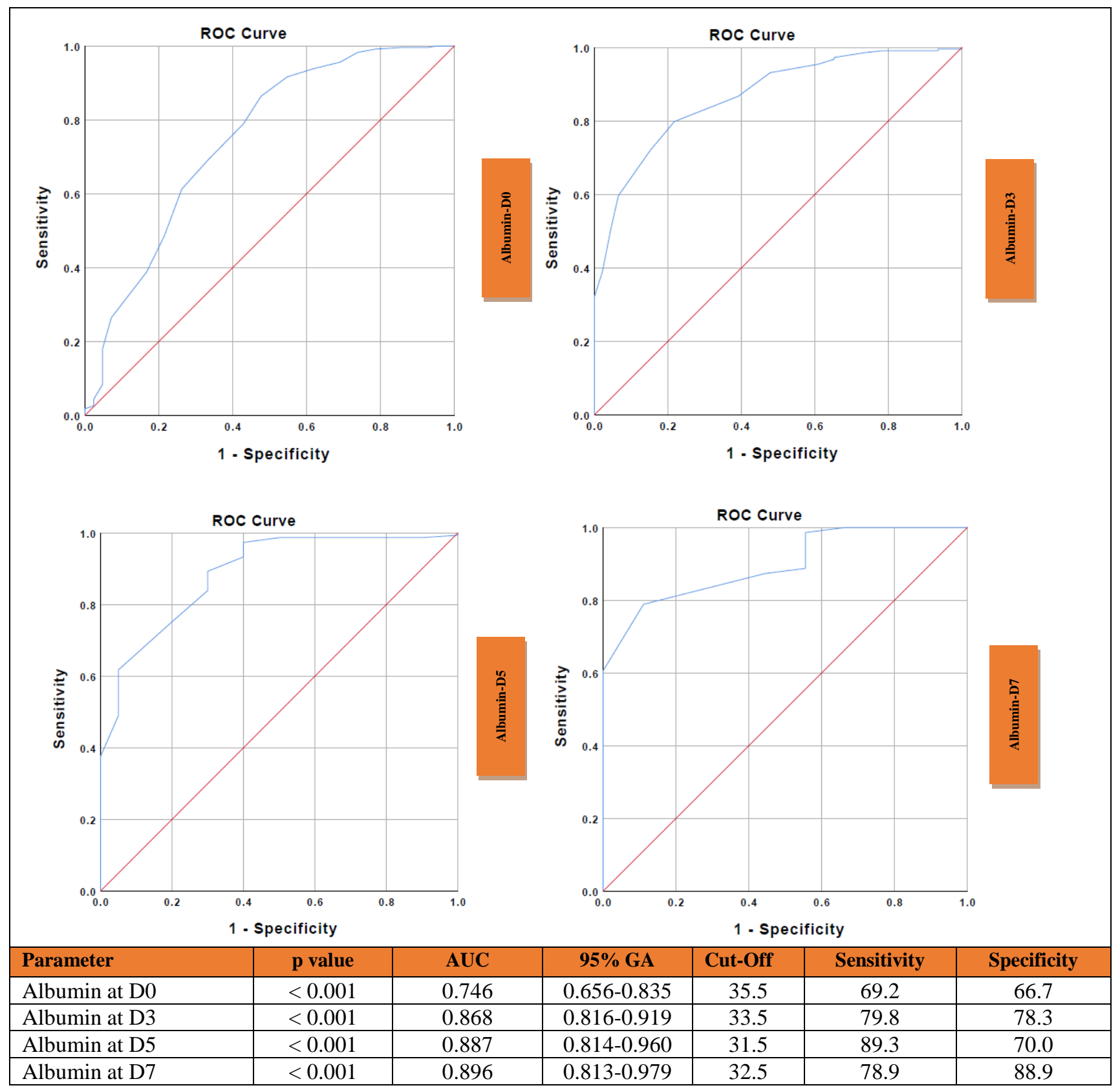


medRxiv preprint doi: https://doi.org/10.1101/2021.01.17.21249878; this version posted January 20, 2021. The copyright holder for this preprint (which was not certified by peer review) is the author/funder, who has granted medRxiv a license to display the preprint in perpetuity.

It is made available under a CC-BY-NC-ND 4.0 International license .

Figure 3. Receiver Operating Characteristic (ROC) curve and performance value for the best cut off for: A: albumin at admission using clinical deterioration B: albumin at D3 using clinical deterioration C: albumin at D5 using clinical deterioration D: albumin at D7 using clinical deterioration.

ROC curves of neutrophil/lymphocyte ratio at D0, D3, D5 and D7 to predict clinical deterioration are shown in Fig. 4. At D0, AUC curve was 0.752 (95\% CI 0.694- 0.810), with the best cut-off of 40.95, sensitivity of $64.5 \%$ and specificity of $77 \%$ (p<0.001). At D3, AUC curve was 0.893 (95\% CI $0.843-0.942$ ), with the best cut-off of 38.60 , sensitivity of $86.7 \%$ and specificity of $81.3 \%$ ( $<<0.001)$. At D5, AUC curve was 0.939 (95\% CI 0.899-0.979), with the best cut-off of 58.6, sensitivity of $79.2 \%$ and specificity of $94.8 \%(\mathrm{p}<0.001)$. At D7, AUC curve was 0.911 (95\% CI $0.834-0.988$ ), with the best cut-off of 58.45, sensitivity of $81.3 \%$ and specificity of $95 \%(\mathrm{p}<0.001)$.
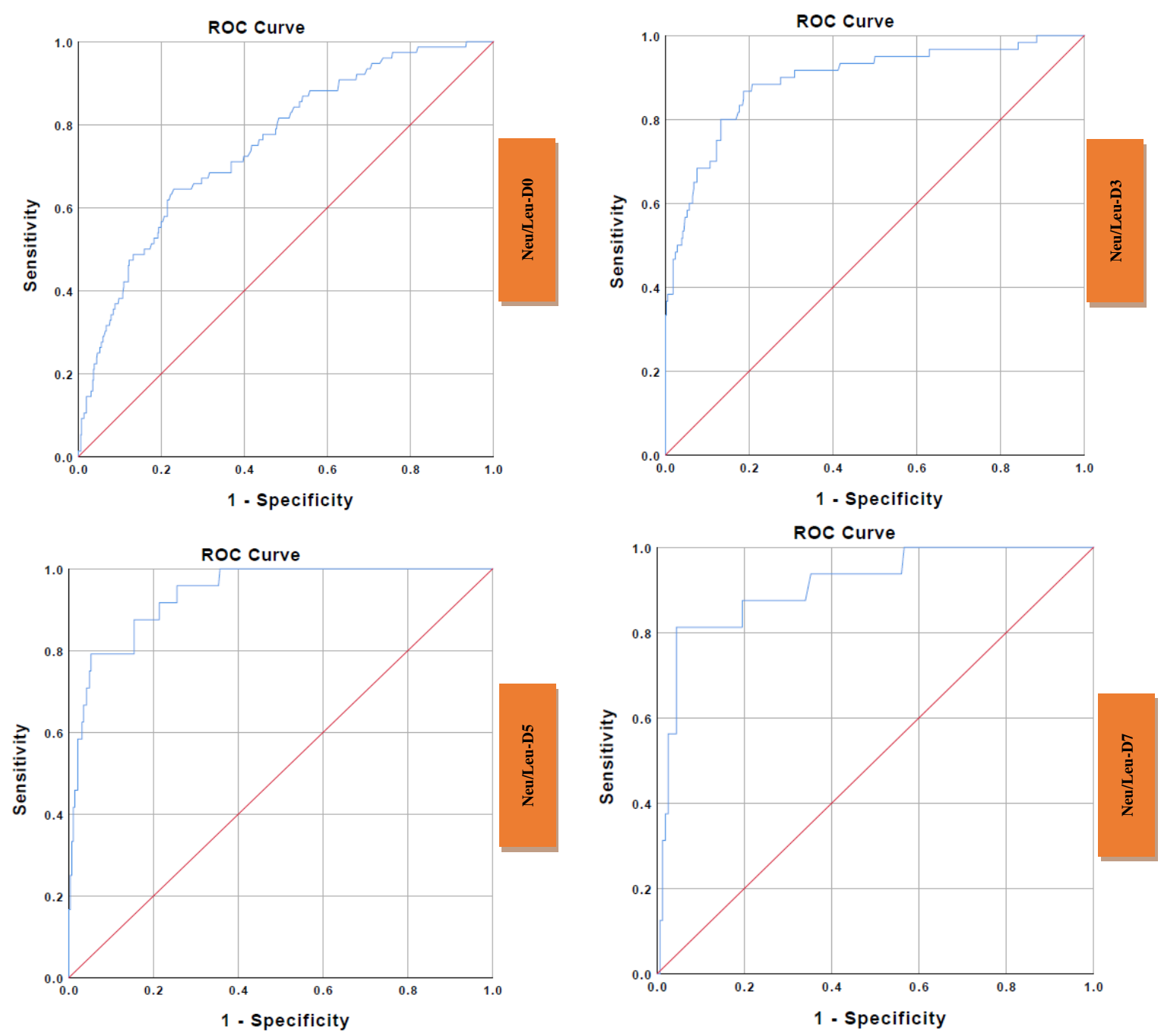
medRxiv preprint doi: https://doi.org/10.1101/2021.01.17.21249878; this version posted January 20, 2021. The copyright holder for this preprint (which was not certified by peer review) is the author/funder, who has granted medRxiv a license to display the preprint in perpetuity.

It is made available under a CC-BY-NC-ND 4.0 International license .

\begin{tabular}{|l|c|c|c|c|c|c|}
\hline Parameter & p value & AUC & 95\% CI & Cut-Off & Sensitivity & Specificity \\
\hline Neutrophil/Lymphocyte at D0 & $<0.001$ & 0.752 & $0.694-0.810$ & 40.95 & 64.5 & 77.0 \\
\hline Neutrophil /Lymphocyte at D3 & $<0.001$ & 0.893 & $0.843-0.942$ & 38.60 & 86.7 & 81.3 \\
\hline Neutrophil /Lymphocyte at D5 & $<0.001$ & 0.939 & $0.899-0.979$ & 58.6 & 79.2 & 94.8 \\
\hline Neutrophil/Lymphocyte at D7 & $<0.001$ & 0.911 & $0.834-0.988$ & 58.45 & 81.3 & 95.0 \\
\hline
\end{tabular}

Figure 4. Receiver Operating Characteristic (ROC) curve and performance value for the best cut off for: A: neutrophil/lymphocyte ratio (NLR) at admission using clinical deterioration B: NLR at D3 using clinical deterioration C: NLR at D5 using clinical deterioration D: NLR at D7 using clinical deterioration

\section{DISCUSSION}

In this study, we presented a detailed analysis of the NEWS2 score and laboratory parameters in hospitalized patients with COVID-19. Our results showed that NEWS2, procalcitonin, NLR, and albumin were the best predictors for clinical deterioration (ICU admission or in-hospital death) at D0, D3, D5, and D7. Procalcitonin had the highest odds ratio for clinical deterioration at D0, D3, D5, and D7 in univariate analysis. ROC analyses showed that NEWS2 at D7, procalcitonin at D5, albumin at D7, and NLR at D5 had highest AUC values. Additionally, we detected a strong correlation between NEWS2 and laboratory parameters including lymphocyte count, neutrophil count, NLR, PLR, CRP, procalcitonin, ferritin, and urea at D0, D3, D5, and D7.

Early and accurate discrimination of need for ICU improves the clinical course of COVID-19 and reduce unnecessary use of ICU beds. To date limited data exists on the NEWS2 and laboratory parameters in patients with COVID-19. There are several published studies on the use of NEWS2 in COVID-19 patients (2-4, 7-12). However, most studies evaluate NEWS2 at admission only (10-13). In the study of Sze et al., they suggested that NEWS2 score was not a valuable tool to predict clinical deterioration in elderly patients with COVID-19 (14). However, they reported the results of only 17 elderly patients. Kim et al. showed that NEWS2 scores on D0 significantly differed in non-critical and critical patients $(2.6 \pm 2.6$ vs. $8.2 \pm 3.3, p<0.001)(9)$. In the study of Volff et al., the AUC value of NEWS score to predict ICU admisson or death was 0.74, in consistent with our result. However, the AUROC values of NEWS2 at D3, D5, and D7 were $0.798,0.833$, and 0.842 whereas NEWS2 at D0 was not accurate (AUROC $<0.750)(7)$. Similarly, Sixt et al. showed that AUROC values of NEWS2 was 0.74 at D0, with a best cut-off of 6 and was 0.98 at D7, with a best cut-off of 7 . They reported high sensitivity and specificity at D7 (\%92 and \%97, respectively) (8). 
Due to the physiopathological changes, deterioration in different laboratory parameters occurs while the disease progresses. Therefore, laboratory parameters are commonly used for assessing disease severity. Lagadinou et al. found an association between COVID-19 severity and the following laboratory parameters NLR, LDH, d-dimers, CRP, fibrinogen, and ferritin (15). In the study of $\mathrm{Xu}$ et al., procalcitonin, CRP, and NLR were valuable predictors for COVID-19 mortality. They showed that the AUC from highest to lowest was combined effect $>\mathrm{CRP}>$ procalcitonin $>$ NLR, respectively (16). Liao et al. reported that NLR, thrombocytopenia, prothrombin time, and D-dimer were associated with death. They showed that increased NLR ( $\geq 9 \cdot 13)$ was associated with 5-fold increased mortality risk (17). Similarly, in our study, increased NLR was associated with OR $=1.286$-fold at D0, OR $=1.754$-fold at D3, OR = 1.806-fold at D5, and OR $=1.332$-fold at D7 increased mortality risk in univariate analysis. In a meta-analysis, Elshazli et al. demonstrated that higher levels of leukocyte $(\mathrm{OR}=5.21)$, neutrophil $(\mathrm{OR}=6.25)$, D-dimer $(\mathrm{OR}=4.19)$, and prolonged PT $(\mathrm{OR}=2.18)$ was associated with ICU admission. IL-6 (OR = 13.87), CRP (OR = 7.09), D-dimer (OR = 6.36), and neutrophils $(\mathrm{OR}=6.25)$ had the highest OR for mortality (18). In a meta-analysis, Lippi et al showed that increased procalcitonin values are associated with a nearly 5-fold higher risk for need for ICU or use of mechanical ventilation (OR, 4.76; 95\% CI, 2.74-8.29) (19). Xu et al. found that procalcitonin ( $\geq 0.10 \mathrm{ng} / \mathrm{mL}, \mathrm{HR}=12.82), \mathrm{CRP}(\geq 52.14 \mathrm{mg} / \mathrm{L}, \mathrm{HR}=12.30)$, and NLR ( $\geq 3.59, \mathrm{HR}=8.6$ ) had higher $\mathrm{HRs}$ of $12.82,12.30$ and 8.6 for mortality, respectively. Additionally, procalcitonin $(\geq 0.10 \mathrm{ng} / \mathrm{mL})$ and CRP $(\geq 52.14 \mathrm{mg} / \mathrm{L})$ but not NLR exhibited independent increasing risks of mortality, with HRs of 52.68 (95\% CI: 1.77-1571.66) and 5.47 (95\% CI: 1.04-28.72), respectively (16).

In the study of Shang et al., Spearman's rank correlation analysis revealed that leukocyte, neutrophil, CRP, procalcitonin, and LDH were positively correlated and albumin was negatively correlated with mortality in patients with receiving maintenance hemodialysis. Additionally, they showed that CRP had the highest AUROC value (0.895), and the values of AUROC of neutrophil count, LDH, leukocyte, albumin, and procalcitonin were $0.813,0.758,0.757,0.743$, and 0.728 , respectively (20). In contrast, we found that procalcitonin was the best predictor for clinical deterioration our study. The optimal cut-off value of procalcitonin at D0, D3, D5, and D7 were $0.065 \mathrm{ng} / \mathrm{mL}, 0.125 \mathrm{ng} / \mathrm{mL}, 0.155 \mathrm{ng} / \mathrm{mL}$, and $0.120 \mathrm{ng} / \mathrm{mL}$, and the sensitivity and 
specificity to predict clinical deterioration were $81.1 \%$ and $67.9 \%$ on $\mathrm{D} 0,80.6 \%$ and $89.3 \%$ on D3, $87.5 \%$ and $96.7 \%$ on D5, and $85.7 \%$ and $88.2 \%$ on D7 respectively.

Procalcitonin is not well studied for COVID-19 cases. However, some studies suggested that increased procalsitonin levels were found to be associated with the disease severity in patients with COVID-19. A meta-analysis showed that severe patients with COVID-19 had increased procalcitonin levels $(18,19)$. Similarly, we found that procalcitonin was the best prognostic parameter for the clinical deterioration in our study. Elevated procalcitonin levels could be associated with acute secondary bacterial pnemonia or systemic secondary bacterial infection in patients with COVID-19 due to the production and release into the circulation from procalcitonin producing extrathyroidal tissues. (21) In our study, despite elevated procalcitonin levels, this elevation was limited. In a previous study by $\mathrm{Xu}$ et al., they suggested that a limited increase in procalcitonin levels could be associated with increased interferon-gamma (16).

Low serum albumin in studies with COVID-19 patients levels are suggested to be associated with an increased risk of mortality (22-25) In consistent with other studies, our results confirm that albumin is a valuable predictor for ICU admission or in-hospital death. Albumin is a negative acute phase reactant produced in the liver and causes down-regulation of the expression of ACE-2 receptors which play a role in the cell entry mechanism of SARS-CoV-2. Liu et al. reported that albumin was associated with clinical deteroration and significantly higher in patients with the improvement/stabilization than in those with disease progression $(36.62 \pm 6.60$ vs. $41.27 \pm 4.55 \mathrm{~g} / \mathrm{L}, \mathrm{p}=0.006)(24)$. In the study of Aziz et al., mean albumin at D0 was $3.50 \mathrm{~g} / \mathrm{dL}$ (CI 3.26-3.74 g/dL) in severe group and 4.05 g/dL (CI 3.82-4.27 g/dL) in non-severe group ( $\mathrm{p}<0.001)$. They reported that hypoalbuminemia was associated with 12.6-fold increased risk of mortality (25).

An increase in neutrophils and a decrease in lymphocytes have been found in various studies. Some studies have shown that NLR may be an important indicator for the severity of COVID -19 patients. Yan et al. showed that NLR was significantly correlated with all-cause in-hospital mortality (OR 44.351; 95\% CI 4.627-425.088) (26). The NLR reflects the balance between the innate and adaptive immune systems (30) and increased NLR levels were found to be associated with clinical deterioration in COVID-19 (16). 
This study has also several limitations. First, it was retrospectively conducted in a single-center. Second, this study had a small sample size and a control group was not included. The generalizability of our results may be limited. Thus, we need new large scale studies providing important information to better understand COVID-19 pandemic. Our study has also several strengths. First, we were able to admit all critically ill patients requiring intensive care to the ICU during the first months of pandemic. This prevent a selection bias. Second, longitudinally evaluation of the association between clinical deterioration and the dynamic changes of laboratory parameters was performed, since we regularly monitored laboratory parameters during the clinical course.

\section{Conclusion}

This study provides a list of several laboratory parameters correlated with NEWS2 and potential predictors for ICU admission or in-hospital death during the clinical course of COVID-19. NEWS2, procalcitonin, NLR, and albumin have a high accuracy to predict clinical outcomes/disease progression in hospitalized patients and should be considered in the clinical decision of ICU admission. In conclusion, dynamic monitoring of NEWS2 and laboratory parameters is vital for improving clinical outcomes.

\section{Acknowledgements}

The authors acknowledge all healthcare professionals who contribute to the care of our patients.

\section{Funding}

This research did not receive any specific grant. No funding was used.

\section{Conflict of Interest}

The authors declare that they have no competing interests.

\section{Author Contributions}

GT proposed the concept, designed the study, wrote the protocol, and managed the study. GT, SS, IYN, and MY, performed the statistics, interpreted the data, and wrote the manuscript. GT, 
SS, HKK, AB, AKC, BC, EZ, GT, HA, IYN, MY, YK were involved in collecting the data. MMS, SSY, FP, GS performed a critical review of the manuscript. All authors provided inputs for revision of the manuscript. SS communicated with the journal and addressed comments from reviewers. All authors contributed to data acquisition, data analysis, or data interpretation, and reviewed and approved the final version.

\section{Ethics Statement}

All procedures performed in studies involving human participants were in accordance with the ethical standards of the Ethics Committee of Haseki Training and Research Hospital and national research committee and with the ethical standards of the Declaration of Helsinki. Written informed consent was waived, given the retrospective nature of this study.

\section{References}

1) Grasselli G, Zangrillo A, Zanella A, et al.; COVID-19 Lombardy ICU Network. Baseline Characteristics and Outcomes of 1591 Patients Infected With SARS-CoV-2 Admitted to ICUs of the Lombardy Region, Italy. JAMA. 2020; 323(16): 1574-81.

2) Scott LJ, Redmond NM, Tavaré A, Little H, Srivastava S, Pullyblank A. Association between National Early Warning Scores in primary care and clinical outcomes: an observational study in UK primary and secondary care. Br J Gen Pract. 2020 May 28; 70(695): 374-80.

3) Greenhalgh T, Treadwell J, Burrow R. NEWS (or NEWS2) score when assessing possible COVID-19 patients in primary care. Cent Evid-Based Med Nuffield Dep Prim Care Health Sci Univ Oxf. 2020; 20.

4) Royal College of Physicians. National Early Warning Score (NEWS) 2: Standardising the assessment of acute-illness severity in the NHS. Updated report of a working party. London: RCP; 2017.

5) Smith GB, Prytherch DR, Meredith P, Schmidt PE, Featherstone PI. The ability of the National Early Warning Score (NEWS) to discriminate patients at risk of early cardiac arrest, unanticipated intensive care unit admission, and death. Resuscitation. 2013; 84(4): 465-70. 
medRxiv preprint doi: https://doi.org/10.1101/2021.01.17.21249878; this version posted January 20, 2021. The copyright holder for this preprint (which was not certified by peer review) is the author/funder, who has granted medRxiv a license to display the preprint in perpetuity. It is made available under a CC-BY-NC-ND 4.0 International license .

6) Zhou F, Yu T, Du R et al. Clinical course and risk factors for mortality of adult inpatients with COVID-19 in Wuhan, China: a retrospective cohort study. The Lancet. 2020 Mar 28; 395(10229): 1054-62.

7) Volff M, Tonon D, Bourenne J, Simeone P, Velly L. No added value of the modified NEWS score to predict clinical deterioration in COVID-19 patients. Anaesth Crit Care Pain Med. 2020; 39(5): 577-8.

8) Sixt T, Moretto F, Devilliers H, et al. The usefulness of NEWS2 at day 7 of hospitalization in predicting COVID-19 evolution and as an early endpoint in therapeutic trials. J Infect. 2020: S0163-4453(20)30639-3.

9) Kim EJ, Hong HL. Letter to the Editor: Discussion of the Article "Prognostic Accuracy of the SIRS, qSOFA, and NEWS for Early Detection of Clinical Deterioration in SARSCoV-2 Infected Patients". J Korean Med Sci. 2020; 35(30): 274.

10) Gidari A, De Socio GV, Sabbatini S, Francisci D. Predictive value of National Early Warning Score 2 (NEWS2) for intensive care unit admission in patients with SARSCoV-2 infection. Infect Dis (Lond). 2020; 52(10): 698-704.

11) Jang JG, Hur J, Hong KS, Lee W, Ahn JH. Prognostic Accuracy of the SIRS, qSOFA, and NEWS for Early Detection of Clinical Deterioration in SARS-CoV-2 Infected Patients. J Korean Med Sci. 2020; 35(25): 234.

12) Myrstad M, Ihle-Hansen H, Tveita AA et al. National Early Warning Score 2 (NEWS2) on admission predicts severe disease and in-hospital mortality from Covid-19- a prospective cohort study. Scand J Trauma Resusc Emerg Med. 2020; 28(1): 66.

13) Goulden R, Hoyle MC, Monis J et al. qSOFA, SIRS and NEWS for predicting inhospital mortality and ICU admission in emergency admissions treated as sepsis. Emerg Med J. 2018; 35(6): 345-9.

14) Sze S, Pan D, Williams CML et al. Letter to the Editor: Variability but not admission or trends in NEWS2 score predicts clinical outcome in elderly hospitalised patients with COVID-19. J Infect. 2020: S0163-4453(20)30341-8.

15) Lagadinou M, Salomou EE, Zareifopoulos N, Marangos M, Gogos C, Velissaris D. Prognosis of COVID-19: Changes in laboratory parameters. Infez Med. 2020; 28(suppl 1): 89-95. 
16) $\mathrm{Xu} \mathrm{JB}, \mathrm{Xu} \mathrm{C}$, Zhang RB, Wu M et al. Associations of procalcitonin, C-reaction protein and neutrophil-to-lymphocyte ratio with mortality in hospitalized COVID-19 patients in China. Sci Rep. 2020; 10(1): 15058.

17) Liao D, Zhou F, Luo L et al. Haematological characteristics and risk factors in the classification and prognosis evaluation of COVID-19: a retrospective cohort study. Lancet Haematol. 2020; 7(9): 671-8.

18) Elshazli RM, Toraih EA, Elgaml A et al. Diagnostic and prognostic value of hematological and immunological markers in COVID-19 infection: A meta-analysis of 6320 patients. PLoS One. 2020; 15(8): e0238160.

19) Lippi G, Plebani M. Procalcitonin in patients with severe coronavirus disease 2019 (COVID-19): A meta-analysis. Clin Chim Acta. 2020; 505: 190-1.

20) Shang W, Li Y, Li H et al. Correlation between laboratory parameters on admission and outcome of COVID-19 in maintenance hemodialysis patients. Int Urol Nephrol. 2020:15.

21) Lippi G, Plebani M. Laboratory abnormalities in patients with COVID-2019 infection. Clin Chem Lab Med. 2020; 58(7): 1131-4.

22) Xu L, Liu J, Lu M, Yang D, Zheng X. Liver injury during highly pathogenic human coronavirus infections. Liver Int. 2020; 40(5): 998-1004.

23) Ponziani FR, Del Zompo F, Nesci A et al.; “Gemelli against COVID-19” group. Liver involvement is not associated with mortality: results from a large cohort of SARS-CoV2-positive patients. Aliment Pharmacol Ther. 2020; 52(6):1060-8.

24) Liu W, Tao ZW, Wang L et al. Analysis of factors associated with disease outcomes in hospitalized patients with 2019 novel coronavirus disease. Chin Med J (Engl). 2020; 133(9): 1032-8.

25) Aziz M, Fatima R, Lee-Smith W, Assaly R. The association of low serum albumin level with severe COVID-19: a systematic review and meta-analysis. Crit Care. 2020; 24(1): 255.

26) Yan X, Li F, Wang X, et al. Neutrophil to lymphocyte ratio as prognostic and predictive factor in patients with coronavirus disease 2019: A retrospective cross-sectional study. $J$ Med Virol. 2020; 92(11): 2573-81. 